\begin{tabular}{|c|c|c|c|}
\hline Eiszeitalter u.Gegenwart & $\mathbf{3 0}$ & $\begin{array}{c}237-252 \\
5 \mathrm{Abb} ., 1 \mathrm{Tab} .\end{array}$ & Hannover 1980 \\
\hline
\end{tabular}

\title{
Der Anstieg des Ostseespiegels im deutschen Küstenraum seit dem Mittelatlantikum
}

\author{
HeINZ KLUG *) \\ Classification, transgression, peat, genesis, sea level, regression, C-14 dating, Holocene,
coastal plain. \\ Baltic Sea, Denmark, German Lowlands, Schleswig-Holstein, Niedersachsen, \\ Bez. Rostock, Bez. Schwerin
}

K urzf a ssung: Nach neuen Untersuchungen zur jungholozänen Entwicklung im Küstenraum der südwestlichen Ostsee ist der Meeresspiegel dort klar phasenhaft in Undulationen und nicht kontinuierlich angestiegen. Dies zeigt eine neue Transgressionskurve, die nach morphologischen Feldarbeiten, archäologischen Befunden, Pollenanalysen und 14C-Datierungen gezeichnet wurde und den Zeitraum der letzten 6000 Jahre erfaßt. Für die Litorina-Zeit können drei Transgressionsphasen (vor 6000 bis 5700 B.P., 5200 bis 4000 B.P., 2900 bis 2000 B.P.), getrennt durch zwei Phasen der Stagnation oder Regression, unterschieden werden. Die postlitorina-zeitliche Entwicklung beginnt mit einem Absinken des Meeresspiegels, worauf ein in Oszillation verlaufender Anstieg zum heutigen Niveau folgt. Transgressive Phasen beginnen um 90 A. D., während der ersten Hälfte des 17. Jahrhunderts und um die Mitte des 19. Jahrhunderts.

Der Vergleich dieser Ergebnisse mit einer neuen stratigraphischen Gliederung der postglazialen Entwicklung im nordöstlichen Küstenraum der DDR zeigt, daß Phasengliederung und -ablauf in beiden Untersuchungsräumen genau übereinstimmen. Die Gleichförmigkeit der Meeresspiegelschwankungen wird glazialeustatisch erklärt, da gegenläufige tektonische Vertikalbewegungen wie mehrmalige Hebungen und Senkungen während der relativ kurzen Entwicklungsperiode auszuschließen sind und die eustatischen Verschiebungen mit klimatischen Schwankungen übereinstimmen.

Abgesehen davon werden die festgestellten Unterschiedlichkeiten in der Höhenlage, die der Meeresspiegel während der Transgressionsphasen erreichte, und die verschiedenen säkularen Anstiegsraten durch isostatischen Einfluß erklärt. Durch die Anwendung einer Methode zur Trennung des isostatischen und eustatischen Anteils an den Bewegungskomponenten können die Abweichungen in regionaler Differenzierung erklärt werden.

Aus einem Vergleich der Ereignisse mit der eustatischen Kurve FaIrbridges (1976) und unter Bezugnahme auf MörnERs (1971) Arbeitsresultate im skandinavischen Hebungsgebiet wird gefolgert, daß beim Anstieg der Ostsee mit der zeitlichen Annäherung an die Gegenwart die eustatische nomponente immer deutlicher hervortritt, während gleichzeitig die Intensität der isostatischen Bewegung abklingt.

\section{[The Transgression in the German Coastal Area of the Baltic Sea since the Middle Atlanticum]}

A bstract: Results of recent investigations on Holocene development in the coastal area of the south-western Baltic Sea have proved that the sea-level in this area has clearly risen to its present level in an oscillating manner rather than continuously. This is shown by a new sea-level curve based on morphological field evidence, archeological results, pollen analyses, and radiocarbon datings, which covers the time interval between $6000 \mathrm{~B}$. P. and the present day. During the Litorina time three phases of transgression (before 6500 to 5700 B.P., 5200 to 4000 B.P., 2900 to 2000 B.P.) seperated by two phases of stagnation or regression can be distinguished. The PostLitorina development begins with a subsiding of sea-level, followed by a rise in an oscillating manner to the present level. Transgressive phases started at about 900 A.D., during the first half of the seventeenth century, and about the middle of the nineteenth century.

*) Anschrift des Verfassers: Prof. Dr. H. Kl u g, Institut für Geographie, Universitätsstr. 31, D-8400 Regensburg. 
The comparison of these results with a new stratigraphic division of the postglacial development in the north-eastern coastal area of the GDR shows that the distinguished phases correspond exactly in both areas studied. The uniformity of the corresponding oscillations of the sea-level is explained by eustatic changes, as tectonic subsidence and uplift during this relativly short period of development can be excluded and as eustatic changes correspond with climatic changes.

In addition, the different heights of sea-level during the transgressive phases and the different secular level changes are explained by isostatic influences. Regional alterations can be explained by using a method for distinguishing isostatic and eustatic components.

A comparison of the results with the eustatic curve of FAIRBRIDGE (1976) and with reference to the results of MörNER's research work (1971) in the Scandinavian area of uplift indicates that the eustatic component of the transgression of the Baltic Sea has become more prominent in present times, while the intensity of the isostatic depression of the area has receded at the same time.

\section{Einleitung}

Die Ergebnisse neuerer Forschungen über den zeitlichen und räumlichen Verlauf der jungholozänen Niveauveränderungen an der Ostseeküste Schleswig-Holsteins (ERNST 1974; Klug 1973 bis 1980; Köster 1971; Voss 1967 bis 1973; vgl. Abb. 1) führen dazu, ein gegenüber älteren Auffassungen (Köster 1960, 1961; Schmitz 1953; TAPfer 1940) revidiertes Bild des Transgressionsvorganges zu zeichnen. Da auch eine neue stratigraphische Gliederung der postglazialen Entwicklung aus dem benachbarten nordöstlichen Küstenraum der DDR (KLIEWE \& JANKE 1978) als Resultat langjähriger Untersuchungen vorliegt, besteht die Möglichkeit, den nacheiszeitlichen Meeresanstieg seit dem mittleren Atlantikum im gesamten deutschen Ostseeküstenraum vergleichend zu verfolgen und daraus wichtige Schlußfolgerungen für die Lösung auch grundsätzlicher Probleme des Zusammenwirkens von Eustasie und Isostasie zu ziehen.

\section{Der Transgressionsverlauf an der schleswig-holsteinischen Ostseeküste}

Der Anstieg des transgredierenden Litorina-Meeres vollzog sich im älteren Holozän zunächst - wie bei prinzipieller Übereinstimmung zwischen allen bisher vorliegenden Untersuchungsergebnissen festgestellt — verhältnismäßig schnell. Divergierende Auffassungen bestehen aber über die Art und das Ausmaß des Meeresanstiegs: erfolgte er kontinuierlich oder oszillierend, lag das Seespiegelniveau schon früh nahe NN oder wurde dieser Stand erst später erreicht?

\subsection{Forschungstand und Grundlagen}

Nach Untersuchungen an den Küsten Ostholsteins - bei Heiligenhafen, im Oldenburger Graben, in der inneren Lübecker Bucht und Alt-Lübeck kam Köster (1960, 1961, 1967) unter Auswertung älterer, vorwiegend pollenanalytisch datierter Transgressionskontakte (TAPFer 1940; Schmitz 1952, 1953) zu dem Ergebnis (vgl. Abb. 2), daß sich im Bereich der südwestlichen Ostseeküste nach einem relativ schnellen Wasseranstieg seit etwa 7500 B.P. der Transgressionsvorgang ab 4500 B.P. zwar verlangsamt, aber insgesamt kontinuierlich fortsetzte. Während diese Annahme sich zunächst (Köster 1960, 1961, 1967) auf den gesamten Zeitraum bis zur Gegenwart bezog, schränkte sie KöstER $(1971,97)$ dann auf die Phase bis Christi Geburt ein und konstatierte, daß der Meeresspiegel von da an oszillierend sein heutiges Niveau erreichte. Altere transgressive Phasen (älter als 2000 Jahre B.P.) sind unbekannt. Grundsätzlich aber wird stets die Auffassung vertreten, daß der Wasserstand bei Christi Geburt noch gut $2 \mathrm{~m}$ unter NN lag. 


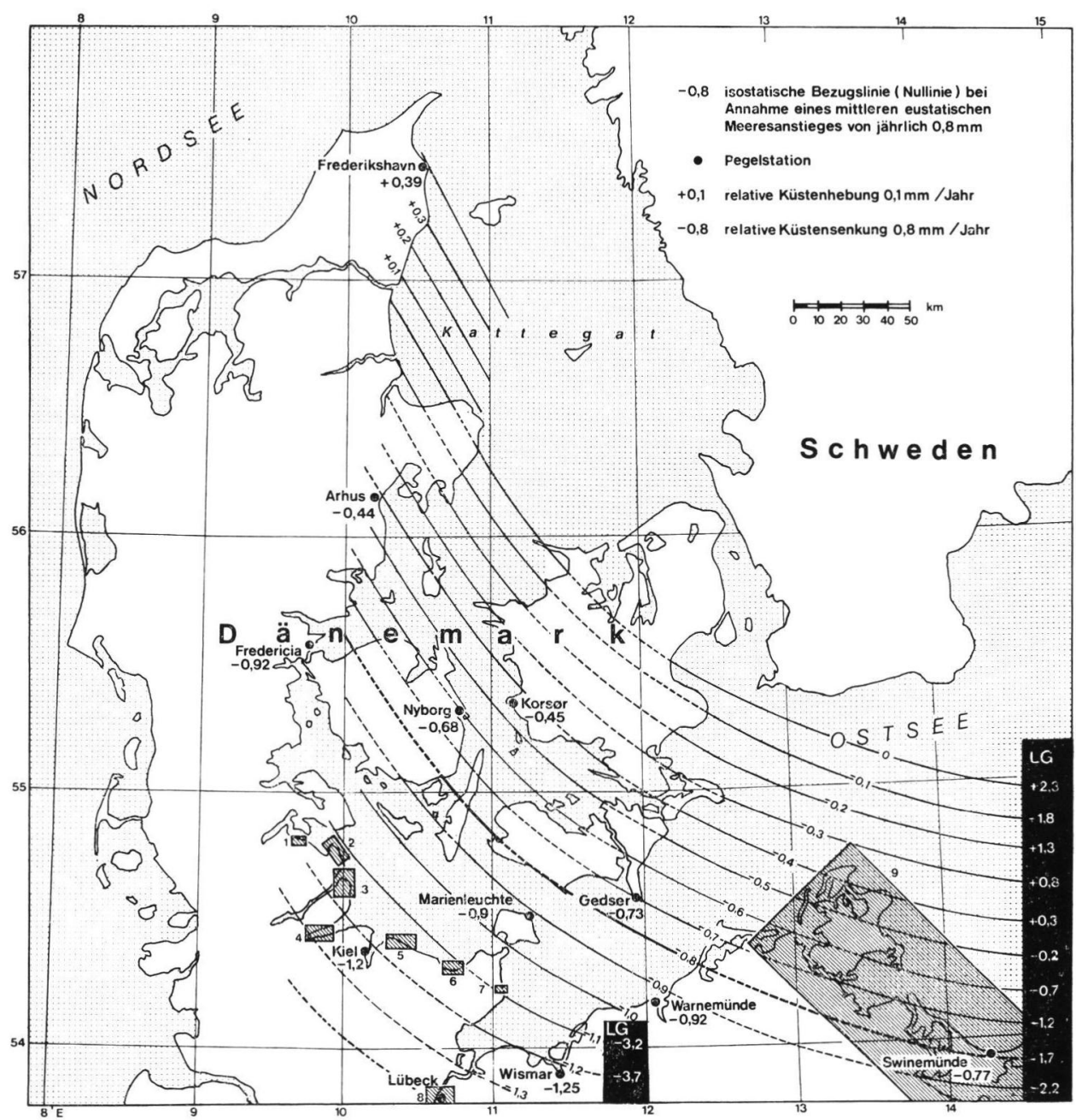

Abb. 1: Ubersichtskarte der südlichen Ostsee mit Isolinien säkularer Pegeländerungen und Angabe der Tiefenlage der Litorina - Grenzfläche (LG) (nach Kolp 1978).

Die schraffierten Flächen zeigen Lage und Größe der neu untersuchten Bereiche: 1 Langballigau, 2 Geltinger Birk, 3 Schleimündung, 4 Eckernförder Bucht, 5 Kieler Außenförde, 6 Hohwachter Bucht, 7 Oldenburger Graben/Süssau, 8 Lübeck, 9 Nordöstl. Küstenraum der DDR.

Entgegen diesen Annahmen zeigen die Ergebnisse der neueren stratigraphischen und küstenmorphologischen Arbeiten aus dem Küstenbereich zwischen Flensburger Förde und Oldenburger Graben, daß der Meeresspiegel schon vor 4000 Jahren nahe bei $-1 \mathrm{~m}$ NN lag und der Transgressionsvorgang insgesamt diskontinuierlich, zeitweise sogar rückläufig, also deutlich oszillierend verlief (vgl. Abb. 2).

Die neuen Erkenntnisse über den postglazialen Meeresanstieg basieren auf geomorphologischen Studien und stratigraphischen Analysen in Verbindung mit modernen Datierungsmethoden. Die Grundlagen für die Erfassung der mit dem Transgressionsvorgang erfol- 


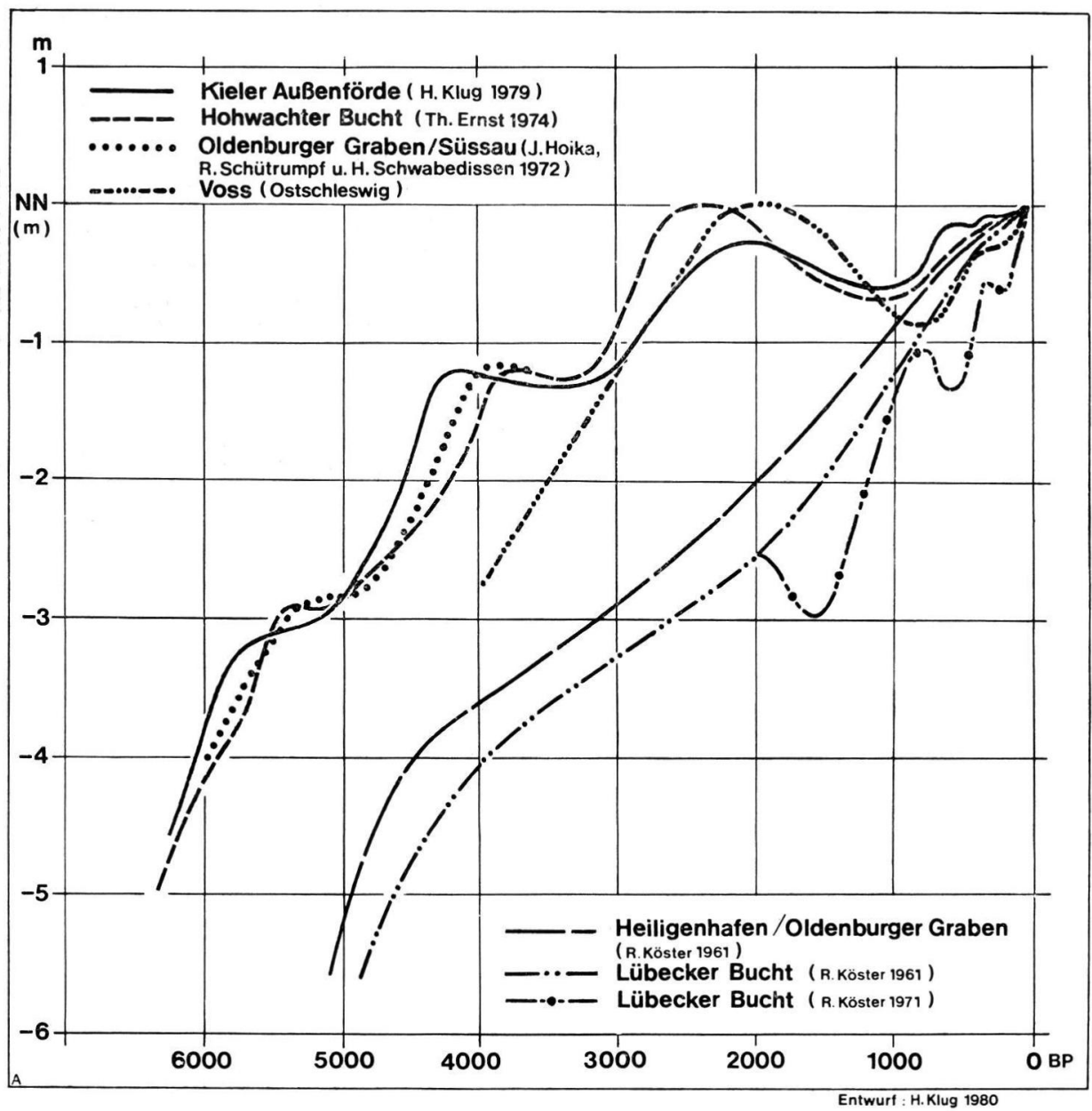

Abb. 2: Transgressionskurven aus dem Küstenraum der südwestlichen Ostsee.

genden Veränderungen der Küstenräume bildeten Aufnahmen der letztglazialen Reliefformen in den terrestrischen und subaquatischen Untersuchungsbereichen. Ausgehend von den heutigen Küstenlandschaften, die durch ausgedehnte Strandwallsysteme geprägt und damit in besonderer Weise auch dem Einsatz geomorphologischer Forschungsmethoden zugänglich sind (Lage s. Abb. 1), wurden - zeitlich zurückschreitend — zunächst unter Anwendung von vermessungstechnischen, kartographischen und geomorphologischen Methoden die einzelnen Phasen der morphologischen Landschaftsgenese rekonstruiert. Die älteren Entwicklungsabschnitte konnten durch die Auswertung von Bohrprofilen, Schürfen und Grabungen, dem daraus resultierenden Sedimentationsablauf sowie aus den Ergebnissen von Pollenanalysen, ${ }^{14} \mathrm{C}$-Datierungen und der chronologischen Einordnung archäologischer Funde in ihrem räumlich-zeitlichen Ablauf erfaßt werden.

Die daraus resultierenden Erkenntnisse sind in Abb. 2 in Form von Transgressionskurven in einem einheitlichen Gitternetz bzw. im gleichen Maßstab dargestellt. Der da- 
durch ermöglichte visuelle Vergleich dieser Ergebnisse aus den verschiedenen Küstenabschnitten der südwestlichen Ostsee dient dem Ziel, sie auf ihre überregionale Aussagekraft hin zu überprüfen. Die vergleichende Betrachtung der Transgressionskurven zeigt eine weitgehende Übereinstimmung sowohl hinsichtlich ihrer Lage innerhalb des Zeit-TiefenDiagrammes als auch bezüglich des oszillierenden Verlaufs.

Eine Abweichung ergibt sich - abgesehen von den Kurven Kösters, auf die noch eingegangen wird - lediglich für das zwischen 4000 und 3000 B.P. verlaufende Teilstück der nach Voss gekennzeichneten Darstellung. Dazu ist zu sagen, daß dieser Kurvenverlauf nur durch einen einzigen Datierungspunkt (-2,80 m um 4000 B.P., südlich der Schleimündung) bestimmt ist, der von TAPFER (1940) pollenanalytisch ermittelt wurde. $\mathrm{Da}$ jedoch ein weiterer Kontakt aus dem Holmer Moor (Schlei) mit -1,25 $\mathrm{m}$ aus derselben Zeit angegeben wird (TAPFER 1940, 196), sind gewisse Zweifel an der pollenanalytischen Aussage und der damit verbundenen Altersstellung des ersten Wertes angebracht (daher das Fragezeichen in Abb. 4), zumal dafür keine Bestätigung durch ${ }^{14} \mathrm{C}$-Messungen erbracht werden konnte. Im übrigen kamen Voss et al. $(1973,79)$ selbst zu dem Ergebnis, daß um 4000 B.P. mit einem höheren Meeresstand zu rechnen ist.

Die in den Kurven zum Ausdruck kommende Ubereinstimmung läßt auf eine entsprechende Gleichförmigkeit des in Undulationen fortschreitenden Meeresspiegelanstiegs in den untersuchten Küstenräumen schließen (KLUG 1980). Damit besteht die Möglichkeit, das zunächst nur mit Gültigkeit für einen jeweils eng begrenzten Raum ermittelte Bild des Transgressionsvorganges auf die Gesamtheit der untersuchten Gebiete mit weiterreichenden Konsequenzen anzuwenden.

$\mathrm{Zu}$ diesem Zweck wurde nach den in den letzten zwölf Jahren erzielten, übereinstimmenden Forschungsergebnissen die neue Kurve des Transgressionsverlaufs für den Küstenbereich der südwestlichen Ostsee entworfen. Abb. 3 zeigt diese Kurve mit der Eintragung aller bisher vorliegenden absoluten Datierungsmarken. Die für jeden Transgressionskontakt bzw. Meßpunkt durch einen Querbalken angegebene zeitliche Schwankungsbreite entspricht dem statistischen Fehlerbereich der ${ }^{14} \mathrm{C}-$ Methode. Da jedoch auch archäologische Zeugnisse, pollenanalytische Datierungen und morphologische Analysen weitere wichtige Zeitmarken für die Bestimmung der relativen Verschiebungen zwischen Land und Meer ergaben, wurden diese zur Dokumentation zusätzlich in Abb. 4 eingetragen. Die Transgressionskurve entspricht der Mittellinie eines Dichtestreifens, in dem sich alle herangezogenen Datierungspunkte anordnen. Nicht zuletzt deshalb, weil viele der Zeitmarken in interdisziplinärer Übereinstimmung festgelegt sind, können auch die Undulationen trotz relativ kurzer Zeitdauer und geringer Amplitude als gut abgesichert gelten. Die ausschließlich pollenanalytisch datierten Transgressionskontakte TAPfERS (1940) aus Ostholstein erwiesen sich beim Vergleich mit neueren ${ }^{14} \mathrm{C}-M e s s u n g e n$ als zu ungenau (vgl. ERNST 1974, 83; Kolp 1976, 22). Diese Angaben wurden deshalb nicht berücksichtigt.

\subsection{Der Entwicklungsgang $z$ wischen Flensburger Förde und Lübecker Bucht}

Wie die Transgressionskurve und Tabelle $1 \mathrm{zu}$ erkennen geben, läßt sich der Meeresspiegelanstieg deutlich in einzelne Phasen gliedern. Der noch im älteren Atlantikum sehr rasche, dann etwas verlangsamte Wasseranstieg (KLIEwe 1978, 88) führte dazu, daß der Ostseespiegel schon im 6. Jahrtausend vor der Gegenwart das Niveau von $-5 \mathrm{~m}$ NN überschritt. Unmittelbar danach erfolgte die Überflutung der Eiszungenbecken, und die Entstehung einer reich gegliederten Buchtenküste begann (ERNST 1974, 79). Damit setzte zugleich die Entwicklung der heutigen Küstenformen ein. 
Diese erste, dem hochlitorina-zeitlichen Abschnitt zuzurechnende Transgressionsphase dauerte bis etwa 5700 B.P. Flächenhafte Vertorfung mit schnellem Höhenwachstum erfolgte zu dieser Zeit am Rand der Buchten und in den Talauen des damaligen Küstenlandes zunächst in dem gleichen Maße (ERNST 1974, 76) wie der Meeresspiegel anstieg. Zunehmend sandiger werdende Tonmudden im Hangenden zeigen dann eine Verstärkung des Transgressionsvorganges gegen Ende dieses Abschnittes an.

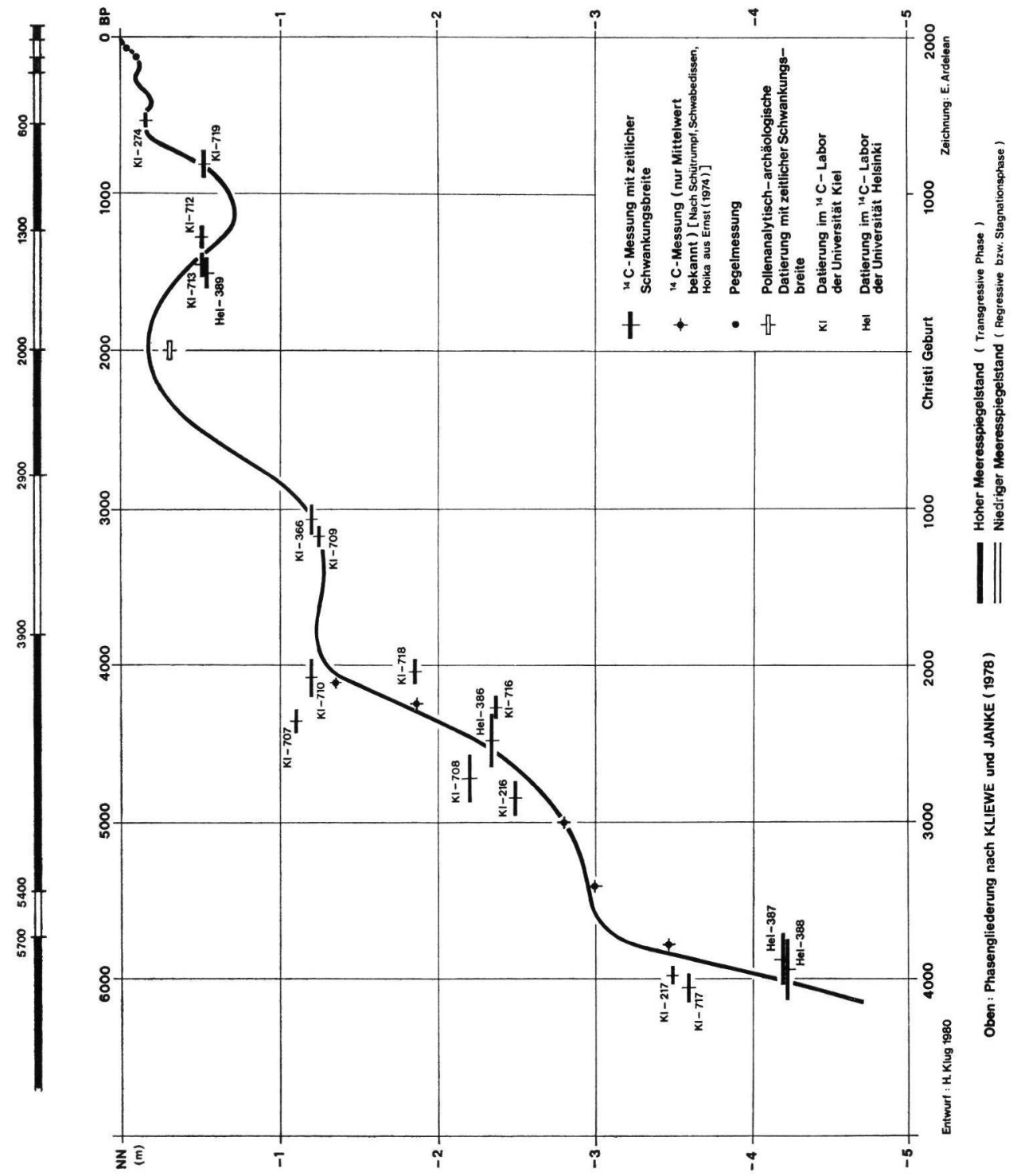

Abb. 3: Transgressionskurve für den Küstenbereich der südwestlichen Ostsee (ausschließlich nach absoluten Datierungen). 


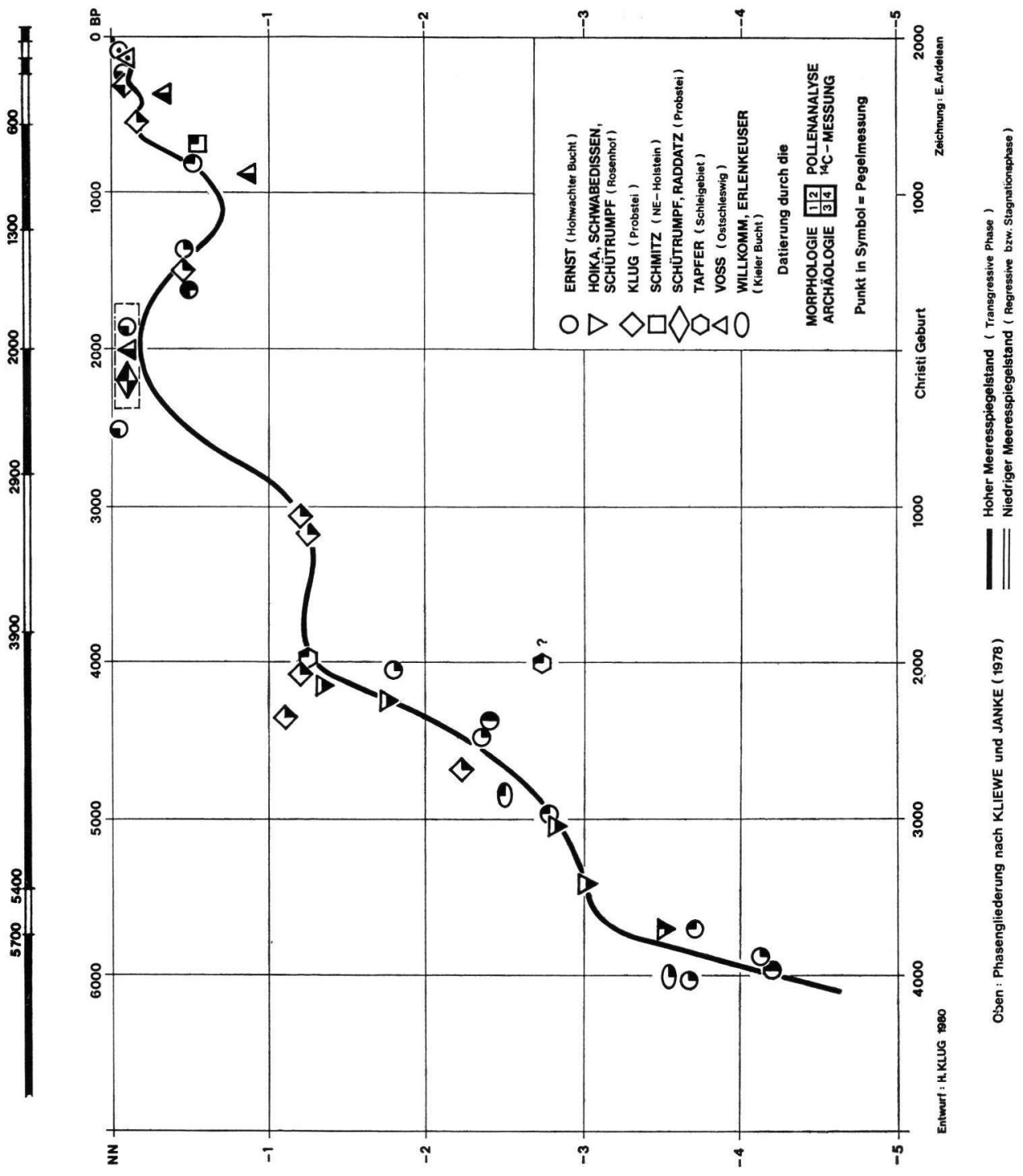

Abb. 4: Transgressionskurve für den Küstenbereich der südwestlichen Ostsee (unter Berücksichtigung aller relevanten Datierungsmarken).

Der Ostseeanstieg wird danach durch eine relativ kurze Phase unterbrochen, in deren Verlauf stark organogene Verlandungssedimente entstehen. Die Transgressionskurve flacht in diesem, von etwa 5700 bis gegen 5200 dauernden Zeitabschnitt stark ab und deutet auf eine Verharrungsphase hin.

Diese Stagnation bildet jedoch nur eine relativ kurze Unterbrechung der Transgression, die von 5200 an zunächst bis etwa 4000 B.P. rasch fortschreitet (2. Abschnitt). In einem 
Jahrtausend steigt der Meeresspiegel von $-2,80 \mathrm{~m}$ (um 5000 B.P.) auf wenige Dezimeter unter $-1 \mathrm{~m}$ an. Das zunächst damit Schritt haltende neuerliche Torfwachstum wird bei fortschreitender Transgression beendet. In den Torf eingelagerte Sandstreifen und -bänder geben die Veränderung der Sedimentationsbedingungen zu erkennen und zeigen das Vor-

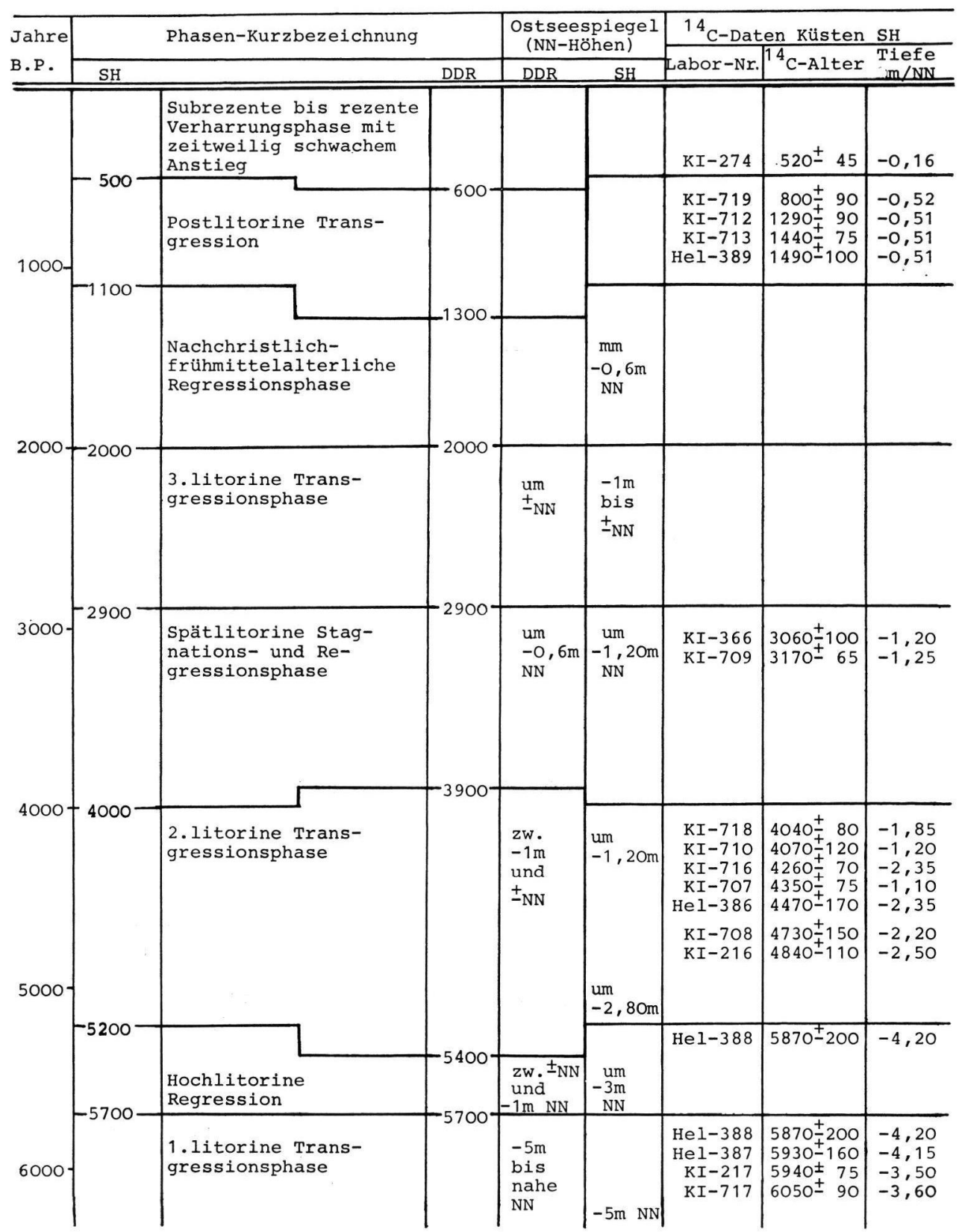

Tab. 1: Phasengliederung der Holozänentwicklung im Ostseeküstenraum Schleswig-Holsteins und der nordöstlichen DDR. 
rücken der Küstenlinie gegen das Festland an. Dieser Transgressionsschub im Ostseeanstieg des jüngeren Holozäns wurde auch bei siedlungshistorischen Untersuchungen (HoIKA 1972, SchütrumpF 1972, Schwabedissen 1972) im Küstenbereich des Oldenburger Grabens und bei Süssau festgestellt. Archäologische, stratigraphische und pollenanalytische Befunde bezeugen die diesbezüglichen Ergebnisse übereinstimmend.

Eine weitere Bestätigung für die Aussage, daß der Meeresspiegel schon vor etwa 4000 Jahren eine Höhenlage von wenigen Dezimetern unter $-1 \mathrm{~m}$ NN erreicht hatte, ergaben naturwissenschaftlich-archäologische Arbeiten an Siedlungsresten des späten Neolithikums bzw. der ältesten Bronzezeit im Küstengebiet von Habernis (BANTELMANN, freundliche mündliche Mitteilung; vgl. auch Voss et al. 1973, 79). Danach stand der Meeresspiegel dort schon etwas früher als 4000 B.P. nicht tiefer als $-1,70 \mathrm{~m}$ im Vergleich zur Gegenwart und war vor 3800 B.P. auf ein mehrere Dezimeter über diesem Wert gelegenes Niveau angestiegen.

Der anschließende Zeitraum von 4000 bis 2900 ist durch ein Verharren des Meeresspiegels im Niveau von wenigen Dezimetern unter $-1 \mathrm{~m}$ NN gekennzeichnet. Diese Stagnations- bis Regressionsphase konnte besonders gut im westlichen Teil der Küstenlandschaft an der östlichen Kieler Außenförde (KLUG 1979) nachgewiesen werden. In einer ruhigen Flachwasserbucht entstanden dort während dieses Zeitabschnittes einerseits Mudden, die von marinen Sanden über- und unterlagert sind, andererseits kam es hinter Strandwällen zu Vermoorung und Torfwachstum. Im oberen Teil der Profile auftretende Sandbänder künden die schließlich einsetzende Überlagerung durch marine Sande und damit einen erneuten verstärkten Meereseinfluß an.

Damit beginnt ein dritter litorina-zeitlicher Transgressionsabschnitt im Küstenraum der südwestlichen Ostsee, der von 2900 bis 2000 B.P. dauert. In seinem Verlauf wird etwa um Christi Geburt ein Ostseespiegelstand erreicht, der fast dem heutigen Normalnull entsprach. Diese Phase der Küstenentwicklung ist gekennzeichnet durch eine starke Uferdynamik mit Höftlandentwicklung sowie dem Aufbau und der Zurückverlegung der ältesten Strandwall- und Hakensysteme. Als Beispiele können die Küstenlandschaften in der Hohwachter Bucht (ERNST 1974: 77) und an der Flensburger Förde (Voss et al. 1973: 78 f.) gelten. Die Morphogenese dieser Hakensysteme leitete gleichzeitig die fortschreitende Abschnürung der Buchten und Talmündungen sowie die Fossilierung zahlreicher zunächst noch aktiver Kliffküsten ein. Infolge der starken transgressiven Vernässung kam es im frühen Subatlantikum noch vor der Zeitenwende in der von der Brandung damals geschützten Küstenniederung der östlichen Probstei (Kieler Außenförde) zu intensivem Torfwachstum (Abb. 5).

Eine neue Phase der Entwicklung setzt um Christi Geburt ein. Sie ist durch ein Zurückweichen des Meeres gekennzeichnet. In dieser, von 2000 bis 1100 B.P. andauernden Regressionsphase sinkt der Ostseespiegel nach dem Kurvenverlauf (Abb. 3 und 4) um etwa einen halben Meter auf ca. $-0,75 \mathrm{~m}$ ab. Datierungsmarken liegen vor für $-0,51 \mathrm{~m}$ um $1290 \pm 90$ B.P., $1440 \pm 75$ B.P. und $1490 \pm 100$ B.P. Auch $800 \pm 90$ B.P. stand der Meeresspiegel noch in diesem Niveau (vgl. Abb. 3), befand sich allerdings wieder im Ansteigen. Denn die nachchristlich-frühmittelalterliche Regressionsphase wurde im frühen 10. Jahrhundert von einer erneut einsetzenden Transgression abgelöst. Der gesamte Entwicklungsgang ist deutlich in Abb. 5 aus der kombinierten Darstellung von Torfwachstum und Meeresspiegelschwankung für ein Grabungsprofil an der östlichen Kieler Außenförde abzulesen.

Morphodynamisch ist dieser Zeitraum durch die Entstehung der jüngeren, zunächst „absteigenden“, dann „aufsteigenden Strandwälle“ charakterisiert, die im Höftland von Langballigau (Voss et al. 1973), in der Geltinger Birk (Voss 1970), an der Schleimündung 
(Voss 1967), der östlichen Kieler Außenförde (KLUG 1973) und in der Hohwachter Bucht (ERNST 1974) untersucht wurden. In jedem dieser Gebiete konnte aus der Topographie der Hakensysteme die Undulation im Transgressionsverlauf abgelesen werden.

Auch der spätmittelalterlich-neuzeitliche Ostsee-Anstieg erfolgte in kleinen Oszillationen, wie die morphologische Analyse der Strandwallsysteme und die Auswertung der Pegelmessungen ergab. Während dieser Phase wurde die Herausbildung von Ausgleichsküsten und die Abschnürung von Strandseen zum wesentlichen Geschehen, das auch heute noch andauert.
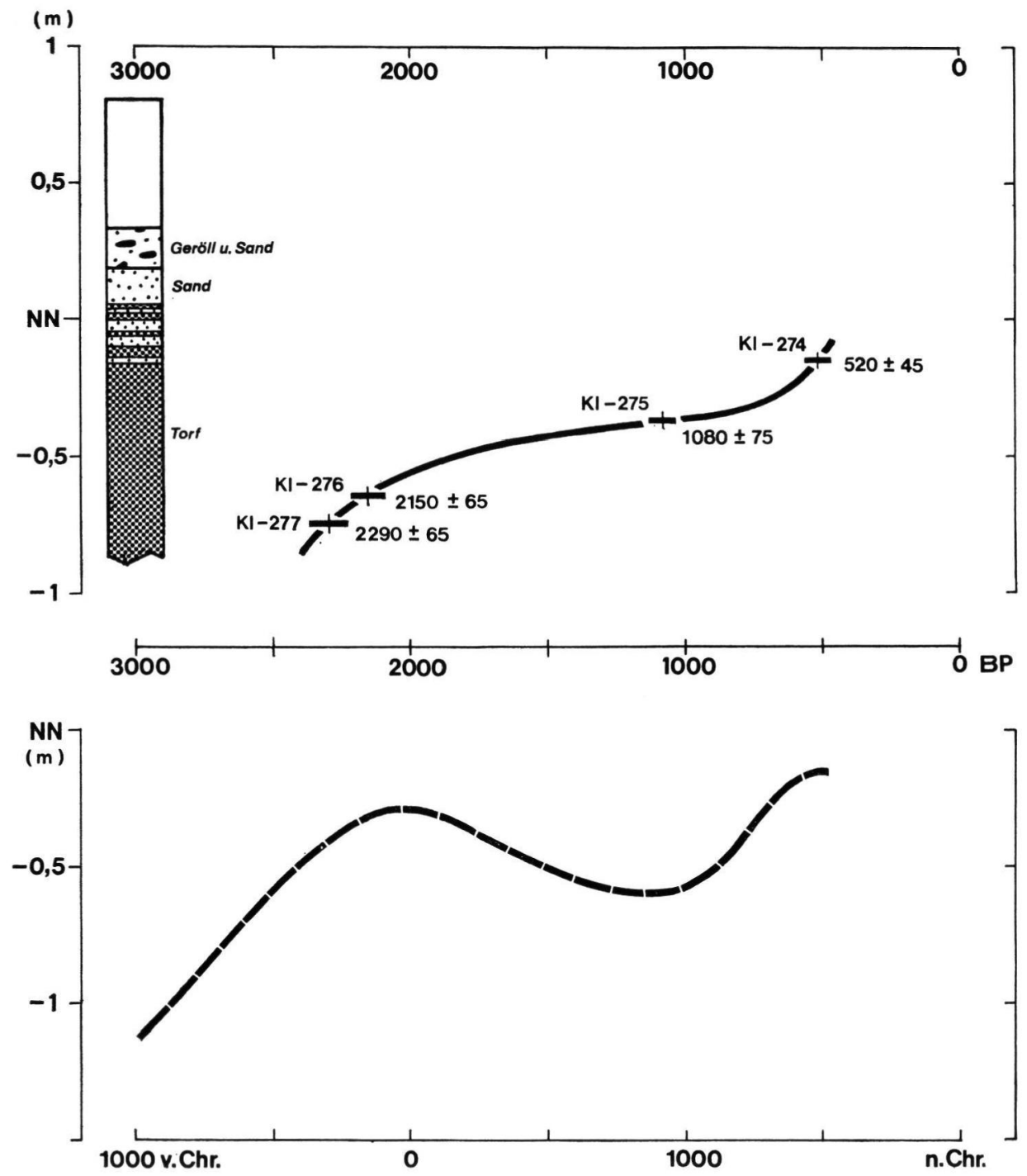

Abb. 5: Relativer Meeresspiegelanstieg und Torfwachstum (Grabung Kalifornien). 
Die postlitorinen Transgressionsphasen fallen jeweils in die Zeiten mit besonders starken Ostsee-Sturmfluten: Die erste nachchristliche Transgression beginnt um das Jahr 900 (1100 B.P.) und wird ab 1200 stärker. In der Mitte des 13. Jahrhunderts betrug die Fläche der Salzwiesen in der nördlichen Probstei (Kieler Außenförde) noch ca. 2000 ha, das sind etwa 600 ha mehr als heute, und die Küste lag noch $0,5 \mathrm{~km}$ weiter seewärts. Eine Sturmflut zerstörte um 1260 das früher dort gelegene Dorf Wisch.

Nach einer Stagnation bzw. Regression nach 1500 A.D. (500 B.P.) beginnt in der ersten Hälfte des 17. Jahrhunderts ein neuerlicher Meeresanstieg. Schwere Sturmfluten sind aus den Jahren 1625 und 1694 bekannt. Im erstgenannten Jahr wurde die „Heide, die nördlich der Probstei lag, überschwemmt und zerstört" (Jessien, in Clasen 1898; DetLefsen 1971: 35 f.). Das ehemals mehr als drei Hufen umfassende Dorf Lippe (Hohwachter Bucht) wurde $\mathrm{zwischen} 1511$ und 1652 durch das vorrückende Meer zerstört und danach nicht mehr genannt (ERNST 1974, 63).

Der jüngste Meeresanstieg erfolgt nach einer vorausgehenden leichten Regression seit Mitte des 19. Jahrhunderts, wie Pegelmessungen und Kartenvergleiche klar zu erkennen geben. In diesen Zeitraum fällt die „Jahrhundert-Sturmflut“ von 1872 (KIECKSEE 1972). Zwischen 1902 und 1968 erfolgte nach den von TRUELSEN (1973) meteorologisch korrigierten und neu berechneten Mittelwerten langjähriger Pegeländerungen ein relativer Meeresspiegelanstieg um $1,0 \mathrm{~mm} / \mathrm{Jahr}$. Die Werte liegen zwischen 0,9 und $1,0 \mathrm{~mm} / \mathrm{Jahr}$ für Marienleuchte und Fehmarnsund sowie 1,2 mm/Jahr für Kiel (vgl. Abb. 1).

\section{Der Vergleich mit der Entwicklung im Küstenraum der DDR}

Die von Kliewe \& JANKE (1978) zusammenfassend dargestellte Entwicklung des Transgressionsverlaufs in dem den schleswig-holsteinischen Untersuchungsgebieten benachbarten nordöstlichen Küstenraum der DDR bildet die Grundlage für einen regionalen Vergleich der zeitlich-räumlichen Verschiebungen zwischen Land und Meer in den beiden Saumregionen des Peribaltikums. Damit besteht zugleich die Möglichkeit, die Gültigkeit der oben getroffenen Aussagen auf ihre überregionale Bedeutung hin zu überprüfen.

Kliewe \& JANKe (1978) gliedern die Darstellung der Entwicklung im nordöstlichen Küstenraum der DDR seit dem Mittelatlantikum in einzelne Zeitabschnitte, für die ein transgressiver oder regressiver bzw. Stagnations-Charakter nachgewiesen werden konnte. Die ermittelten Phasen sind in die Abbildungen 3 und 4 als Zeitabschnitte aufgenommen. Ein schwarz ausgezogener Balken steht für „Transgression“, ein offener für „Regression“ bzw. "Stagnation“.

Die Entwicklung bis zur Zeitenwende wird von KLIEWE \& JANKE (1978: 88 f.) in drei „Litorine-Hauptphasen“ unterteilt. Das sind transgressive Zeitabschnitte zwischen 7000 und 5700 B.P., 5400 und 3900 B.P. sowie 2900 und 2000 B.P. Dazwischen liegt eine "hochlitorina-“ (5700-5400 B.P.) und eine "spätlitorina-zeitliche" (3900-2900 B.P.) Regressionsphase.

Für den postlitorina-zeitlichen Entwicklungsabschnitt werden eine leichte Regression während der römischen Kaiserzeit und der beginnenden Slawenzeit (2000-1300 B.P.) sowie eine postlitorina-zeitliche Transgressionsphase (1300-600 B.P.) wahrscheinlich gemacht. Die subrezente Entwicklung bis in die Gegenwart ist „durch ein erneutes Verharren mit zeitweilig, so auch gegenwärtig, schwachem Anstieg des Ostseespiegels gekennzeichnet" (KLIEWE \& JANKE 1978: 90).

Der relative Meeresspiegelanstieg beträgt im nordöstlichen Küstenraum der DDR nach Montag (1974) und Kolp $(1978,183)$ bei Swinemünde $0,77 \mathrm{~mm} / \mathrm{Jahr}$ und verringert sich nordwärts, so daß er am Nordrand Rügens bei etwa $0,4 \mathrm{~mm} / \mathrm{Jahr}$ liegt (vgl. Abb. 1). 


\subsection{Entwicklungsparallelen: eustatischer Einfluß}

Der Vergleich dieser Phasengliederung KLIEwes \& Jankes (1978) mit dem für die Niveauverschiebungen in den Strandwallandschaften Schleswig-Holsteins herausgefundenen Zeitabschnitten zeigt eine geradezu erstaunliche Übereinstimmung der Untersuchungsergebnisse (vgl. auch Tab. 1). Wo Abweichungen auftreten, betragen sie nie mehr als 100 bis 200 Jahre; sie bleiben damit immer in der Schwankungsbreite des Fehlerbereichs der Datierungsmethoden und beeinträchtigen das Bild der Übereinstimmung nicht. Im einzelnen zeigt auch die vergleichende Betrachtung der Abschnittsgliederung KLIEwES \& JANKES (1978) und des Kurvenverlaufs in den Abbildungen 3 und 4, wie weitgehend sich die verschiedenen Phasen entsprechen.

Nach diesen Darlegungen kann der insgesamt diskontinuierliche, zeitweise sogar rückläufige Vorgang der Transgression schwerlich auf tektonische Verlagerungen zurückgeführt werden. Denn mit großer Wahrscheinlichkeit sind für den betrachteten, relativ kurzen Zeitraum gegenläufige Vertikalverschiebungen, wie sie in einem mehrmaligen Heben und Senken des Küstenraumes zum Ausdruck kämen, auszuschließen. Da andererseits aufgrund der Gleichförmigkeit des nachgewiesenen und in der Kurve dargestellten Transgressionsverlaufes im gesamten Gebiet der südwestlichen Ostsee nur ein überregionaler Faktor für das "Oszillieren“ maßgebend gewesen sein kann, dürften die Undulationen des phasenhaften Meeresspiegelanstiegs der letzten 5000 bis 6000 Jahre in erster Linie eustatisch bedingt sein.

Diese Schlußfolgerung wird dadurch bestätigt, daß sich die eustatischen Verschiebungen des Ostseespiegels zwanglos mit klimatischen Veränderungen in Einklang bringen lassen. Dies gilt sowohl für die litorina-zeitliche Holozänentwicklung als auch für den subrezenten Verlauf. Für die letzten 300 Jahre wurden entsprechende Parallelen durch MöRNER (1973) nachgewiesen.

\subsection{Entwicklungsdivergenzen: isostatischer Einfluß}

Es bleibt festzustellen, daß beim Vergleich des Entwicklungsganges zwischen den Küstenräumen Schleswig-Holsteins und dem NE der DDR auch deutliche Unterschiede auszumachen sind. Diese Divergenzen beziehen sich ausschließlich auf die Lage der Wasserstandsmarken, die für die einzelnen Zeitabschnitte ermittelt wurden. Das Ostseespiegelniveau erreichte nach KLIEWE \& JANKE (1978: 89) im nordöstlichen Küstenraum der DDR schon im jüngeren Atlantikum $-2 \mathrm{~m}$ bis $-1 \mathrm{~m}$ NN. Während der letzten 4000 bis 5000 Jahre betrug dort der oszillierend erfolgende Meeresanstieg bis zum heutigen Stand nur noch einen Meter. An den Küsten Schleswig-Holsteins trat diese Entwicklung mit einer zeitlichen Verzögerung von 500 bis 1000 Jahren erst um 4000 B.P. ein. Daß im Küstenraum der DDR das heutige Meeresspiegelniveau bei sonst übereinstimmendem Ablauf der Entwicklung in den zu vergleichenden Untersuchungsgebieten früher erreicht wurde als an den schleswig-holsteinischen Küsten, kann seine Ursache nur in einer unterschiedlich raschen gleichsinnigen oder einer gegenläufigen vertikalen Eigenbewegung des Festlandes haben, die den eustatischen Meeresanstieg überlagert. Im südlichen bis westlichen Ostseegebiet kommt dafür in erster Linie die isostatische Höhenverschiebung in Frage.

In der Tendenz entspricht dieser Entwicklungsdivergenz das rezente Verhalten des Ostseespiegels in den zu vergleichenden Küstenräumen. Dies veranschaulicht die nach Kolp $(1978,183)$ gezeichnete Karte der südlichen Ostsee mit Isolinien säkularer Pegeländerungen (Abb. 1). In ihrem zueinander parallelen Verlauf zeigt die Kurvenschar eine große Ähnlichkeit sowohl mit den Isobasen der Hebung Fennoskandiens seit Beginn der Litorina-Zeit als auch mit den Linien gleicher Küstensenkung der letzten 2000 bis 3000 Jahre (Köster 1961). In dem vom Nordrand Rügens von $-0,4 \mathrm{~mm} / \mathrm{Jahr}$ auf $1,2 \mathrm{~mm} /$ 
Jahr in Kiel zunehmenden Betrag der Wasserstandserhöhung kommt die bekannte Tatsache zum Ausdruck, daß die relative Küstensenkung in südwestlicher Richtung, d.h. mit der Entfernung vom skandinavischen Hebungsgebiet zunimmt.

Von Bedeutung ist in diesem Zusammenhang die Lage der betroffenen Küstenräume zu der von KösTER (1961) gezeichneten tektonischen Null-Linie. Sie entspricht nach KolP $(1979,181)$ in der von ihm entworfenen Karte der Isolinien säkularer Pegeländerungen der Verbindungslinie derjenigen Küstenstationen, an denen die relative Küstensenkung $0,8 \mathrm{~mm} / \mathrm{Jahr}$ beträgt (vgl. Abb. 1). Da für den weltweiten eustatischen Meeresanstieg ein Mittelwert von ebenfalls 0,8 mm/Jahr errechnet wurde (MonTAG 1974), folgt daraus, daß die relative Senkung an der tektonischen Null-Linie dem rezenten eustatischen Ostseespiegelanstieg entspricht und die isostatische Vertikalbewegung an dieser Linie gleich Null ist.

Sie verläuft durch die Mitte Fünens, südlich von Lolland, am Nordrand der Lübecker Bucht entlang zur Recknitz-Mündung und weiter durch Mecklenburg zum Ostteil der Insel Usedom. Die Küstenräume im NE der DDR liegen demnach im relativen Hebungsgebiet, die Strandwallandschaften Schleswig-Holsteins dagegen im Bereich der Senkung.

Durch die in nördlicher Richtung zunehmende isostatische Hebung erfolgte eine Schrägstellung der von Kolp (1976) als Marken des holozänen Meeresanstiegs beschriebenen „submarinen Uferterrassen“. Aus der Kippung dieser Grenzflächen, die der Spiegelfläche einer früheren Ostseephase entsprechen, entwickelte Kolp (1979) unter Verwendung der tektonischen Null-Linie als Bezugslinie ein Verfahren zur Trennung der eustatischen und isostatischen Komponenten der Vertikalbewegungen. Aus den berechneten isostatischen Beträgen können mit Hilfe eines Koordinatennetzes, in dem die Ordinaten radial zum Hebungszentrum und die als Abzissen dienenden Linien gleicher säkularer Pegeländerungen marginal verlaufen, die isostatischen Beträge für andere Punkte des südlichen Ostseeraumes durch Interpolation festgestellt werden. Die für die Tiefenlage (unter NN) der Litorina-Grenzfläche (LG) (etwa 5000-6000 B.P.) ermittelten Werte sind in der Karte (Abb.1) nach KolP $(1979,183)$ eingetragen. Bei Anwendung dieses Verfahrens auf die Untersuchungsgebiete lassen sich die in der Höhenlage des Meeresniveaus auftretenden Unterschiede im Transgressionsverlauf als isostatisch bedingt erklären. So geht auch aus Abb. 1 hervor, daß etwa an der östlichen Kieler Außenförde, die nahe der Isolinie einer relativen Küstensenkung von $-1,2 \mathrm{~mm} / \mathrm{Jahr}$ liegt, der Pegel vor 5000-6000 Jahren etwas höher als -3,5 m NN stand, was der Seespiegelkurve (Abb. 2, 3 und 4) entspricht. Die weitgehende Übereinstimmung des Transgressionsverlauf in den Küstengebieten Schleswig-Holsteins erscheint nicht zufällig, sondern wird dadurch erklärbar, daß sie alle an derselben Marginallinie (etwa -1,15) liegen (Abb. 1).

Dies trifft jedoch nicht für den Bereich der inneren Lübecker Bucht zu. Die relative Küstensenkung ist hier analog der größeren Entfernung zur tektonischen Null-Linie stärker, so daß die Litorina-Grenzfläche dort in einer Tiefe von etwa $-5 \mathrm{~m} \mathrm{NN}$ liegt. Dieser Wert entspricht der Kurve des Wasseranstiegs bei Alt-Lübeck (Abb. 2, Köster 1961), die aus den gleichen Gründen im Zeit-Tiefen-Diagramm auch unterhalb der für den übrigen schleswig-holsteinischen Ostseeküstenraum gültigen Transgressionskurve liegen muß. Allerdings ist die Frage noch offen, ob hier der Meeresspiegel in der vorchristlichen Zeit tatsächlich ohne Undulationen, also kontinuierlich anstieg.

\section{Ergebnisse und Folgerungen aus einem ubiquitären Vergleich}

Die vergleichende Betrachtung der jungholozänen Entwicklung im Ostseeküstenbereich Schleswig-Holsteins und der nordöstlichen DDR ergab weitgehende Parallelen hinsichtlich des eustatisch bedingten Meeresanstiegs und offenbarte Divergenzen in der isostatischen 
Beeinflussung des Transgressionsverlaufs. Für die regionale Differenzierung der isostatischen Ausgleichsbewegungen erwies sich der Abstand der untersuchten Küstenräume zur tektonischen Null-Linie als entscheidend, da an dieser Linie auch die säkuläre relative Küstensenkung ( $-0,8 \mathrm{~mm} / \mathrm{Jahr}$ ) dem rezenten Meeresanstieg entspricht und sie als Bezugslinie für eine Berechnung der Tiefenlage früherer Ostseestände dienen konnte (KoLP 1979). Auf diese Weise ließen sich örtlich verschiedene Kurven des jungholozänen Meeresanstiegs sowie die unterschiedlichen Beträge der säkularen Pegeländerungen und relativen Küstensenkung in Einklang bringen.

Der glazialisostatische Einfluß auf die jungholozänen Niveauveränderungen ergibt sich schließlich aus einem Vergleich der für das schleswig-holsteinische Küstengebiet erarbeiteten Transgressionskurve mit derjenigen, die FAIRBRIDGE $(1961,1976)$ vorgelegt hat, denn im südwestlichen Ostseegebiet bleibt die absolute Höhenlage des Meeresspiegels trotz eines sehr starken relativen Anstiegs - stets unter den in „stabilen“ Küstenregionen der Erde erreichten Niveaus. Zwar treten beim Vergleich der Kurven vor allem in der älteren Litorina-Zeit einzelne, zunächst nicht zu erklärende Phasenverschiebungen für Trans- und Regressionsabschnitte auf, doch zeigen die Undulationen der jüngeren Zeit (seit etwa 3500 B.P.) recht gute Übereinstimmungen.

So entspricht der Meeresanstieg, der zu einem Maximum um Christi Geburt führt, bei FAIRBRIDGe $(1978,358)$ der transgressiven "Periode VI“ von 2600 bis 2000 B.P. Zugleich wird festgestellt, daß bereits aus der Zeit von 3000 bis 2700 B.P. Anzeichen für den Beginn dieser Transgressionsphase vorliegen, nachdem der Meeresspiegel vorher von etwa $+3 \mathrm{~m}$ NN auf einen dem heutigen Niveau ähnlichen Stand abgesunken war (= spätlitorina-zeitliche Stagnations- bis Regressionsphase). Die erste Regression und darauf folgende Transgression nach der Zeitenwende liegen in FAIRbridges Kurve von 1976 einige Jahrhunderte früher, stimmen dagegen mit seiner früheren Gliederung (FAIRBRIDGE 1961) zeitlich ziemlich genau überein. Die nachfolgenden Entwicklungsabschnitte entsprechen sich in beiden Darstellungen.

Von besonderer Bedeutung ist beim Vergleich des Transgressionsverlaufs, wie er an der südlichen bis westlichen Ostsee ermittelt wurde, mit der Kurve Fairbridges (1976), daß sich die Undulationen auch im Senkungsgebiet des deutschen Küstenraumes mit der Annäherung an die Gegenwart immer deutlicher ausprägen. Entspricht zunächst (vor 3000 B.P.) der ubiquitären Regression an den Ostseeküsten Schleswig-Holsteins und der nordöstlichen DDR als Folge der Uberlagerung des eustatischen Meeresanstiegs durch die isostatische Landsenkung nur eine Stagnationsphase, so kommt es nach der Zeitenwende hier tatsächlich wiederholt auch zu einer Meeresspiegelsenkung. Während dieses Entwicklungsganges werden gleichzeitig die Differenzen der Amplituden in den Transgressionskurven sowohl bei FAIRBRIDGE (1976) als auch in der hier vorgelegten immer geringer.

$\mathrm{Da}$ sich trotzdem im Senkungsgebiet die eustatisch bedingten Oszillationen auch in Form von Regressionen auswirken, kann daraus gefolgert werden, daß im jungen Holozän die Intensität der isostatischen Bewegungen zur Gegenwart hin immer schwächer werden. Diese Feststellung wird bestätigt durch die von Mörner (1971) entworfenen ZeitGradient-Kurven für die isostatisch beeinflußten Küstenlinien im südlichen Skandinavien. Nach Kolp $(1979,184)$ spricht auch die seit 9000 Jahren fast gleichmäßige Kippung verschiedener submariner Uferterrassen für ein allmähliches Abklingen der isostatischen Vertikalbewegungen im Bereich der südlichen Ostsee.

Eine integrative Auswertung dieser Befunde für die Vertikalbewegungen im Küstenraum der südlichen bis westlichen Ostsee (KLUG 1980) führt zu folgendem Ergebnis: In den Gebieten, in denen der Meeresanstieg von einer Landsenkung überlagert wird, treten mit der zeitlichen Annäherung an die Gegenwart die rein eustatischen Bewegungen, wie 
sie auch aus anderen, „stabilen“ Küstengebieten der Erde bekannt sind, immer deutlicher in Erscheinung. Die Intensität der isostatischen Landsenkung wird während dieses Zeitraumes analog immer schwächer. Der Küstenraum der südwestlichen Ostsee wurde demnach im Holozän nicht von einer einheitlichen, zeitlichen kontinuierlichen Landsenkung betroffen. Sie klingt vielmehr allmählich in dem Maß aus, wie die eustatische Komponente der Bewegungen immer deutlicher hervortritt.

\section{Schriftenverzeichnis}

Clasen, H. (1898): Die Probstei in Wort und Bild. Beitrag Jessien: 9-53; Schönberg i. Holst.

Detlefsen, N. (1971): Schönberg im Wandel der 3 Jahrhunderte von 1600—1900. — Schönberg i. Holst.

ERnst, Th. (1974): Die Hohwachter Bucht. Morphologische Entwicklung einer Küstenlandschaft Ostholsteins. - Schrift. Naturwiss. Ver. Schl.-Holst., 44: 47-96; Kiel. [Diss.].

Fatrbridge, Rhodes W. (1961): Eustatic changes in sea level. - Physics and Chemistry of the Earth; New York.

- (1976): Shellfish-Eating Preceramic Indians in Coastal Brazil. Radiocarbon Dating of shell middens discloses a relationship with Holocene sea level oscillations. - Science, 191: 353359; New York.

Guenther, E. W. (1952): Fundumstände und die Geologie des Moores und seiner Umgebung: $33-37$, s. Guenther et al.

Guenther, E. W., G. Nobis, K. Raddatz \& R. SchütrumpF (1952): Frühgeschichtliche Moorfunde von Barsbek (Kreis Plön). - Meyniana, 1: 32-57; Kiel.

Horka, J. (1972): Süssau, ein neolithischer Wohnplatz an der Ostsee. - Archäologisches Korrespondenzblatt, 2: 17-19; Mainz.

KIECKSEE, H. (1972): Die Ostsee-Sturmflut 1872. - Schriften des deutschen Schiffahrtsmuseums Bremerhaven, 2: $0-0$; Heide.

KLIEwE, H. \& W. JANKE (1978): Stratigraphie und Entwicklung des nordöstlichen Küstenraums der DDR. - Peterm. Geogr. Mitt., 122, 2: 81-91; Gotha/Leipzig.

KLUG, H. (1973): Neue Forschungen zur Küstenentwicklung des südwestlichen Ostseeraumes. Kieler Universitätstage 1973, 10 Vorträge: 101-126, Kiel.

- (1979): Ergebnisse küstenmorphologischer Untersuchungen im Bereich der östlichen Kieler Außenförde und ihre überregionale Bedeutung. - Vortrag in Kiel am 22. 1. 1979.

- (1980): Art und Ursachen des Meeresanstiegs im Küstenraum der südwestlichen Ostsee während des jüngeren Holozän. - Berl. Geogr. Stud., 7 (Hofmeister B. u. A. Steinecke (Hrsg.): Beiträge z. Geomorphologie und Länderkunde, Prof. Dr. Hartmut Valentin zum Gedächtnis), Berlin. (i. Druck)

- , H. Eerlenkeuser, Th. Ernst \& H. Willkomm (1974): Sedimentationsabfolge und Transgressionsverlauf im Küstenraum der östlichen Kieler Außenförde während der letzten 5000 Jahre. - Offa, 31: 5-18; Neumünster.

Kolp, O. (1976): Submarine Uferterrassen der südlichen Ost- und Nordsee als Marken des holozänen Meeresanstiegs und der Überflutungsphasen der Ostsee. - Peterm. Geogr. Mitt., 120, 1:1-23; Gotha/Leipzig.

- (1979): Eustatische und isostatische Veränderungen des südlichen Ostseeraumes im Holozän. - Peterm. Geogr. Mitt., 123, 3: 177-187; Gotha/Leipzig.

Köster, R. (1960): Junge isostatische und eustatische Bewegungen im südlichen und westlichen Ostseeraum. - Neues Jb. Geol. Paläont. Mh.; 70-95; Stuttgart.

- (1961): Junge eustatische und tektonische Vorgänge im Küstenraum der südwestlichen Ostsee. - Meyniana, 11: 23-81; Kiel.

- (1967): Der nacheiszeitliche Transgressionsverlauf an der schleswig-holsteinischen Ostseeküste im Vergleich mit den Kurven des weltweiten eustatischen Wasseranstiegs. - Baltica, 3: 23-41; Vilna.

- (1971): Postglacial Sea-Level Changes on the German Northsea and Baltic Shorelines. Quaternaria, 14: 91-100; Rom.

MörnER, N.-A. (1971): Eustatic Changes during the last 20.000 Years and a Method of seperating the isostatic and eustatic factors in an uplift area. - Palaeogeography, Palaeoclimatol., Palaeoecol., 9: 153-181; Amsterdam. 
MörnER, N.-A. (1973): Eustatic Changes during the last 300 Years. - Palaeogeography, Palaeoclimatol., Palaeoecol., 13:1-14, Amsterdam.

Montag, H. (1974): Zur Bestimmung rezenter Vertikalbewegungen aus langjährigen Registrierungen von Mareographen. - Geodätische und geophysikalische Veröffentl., III, 35: 36-41; Berlin.

Noвis, G. (1952): Die Tierknochen von Barsbek, s. Guenther et al.: 50—57.

Raddatz, K. (1952): Die Keramik des Barsbeker Moorfundes, s. Guenther et al.: 44-49.

Schмiтz, H. (1952): Der pollenanalytische Nachweis der Besiedlung im Küstengebiet. - Abh. naturw. Ver. Bremen, 33: 57-66; Bremen.

- (1953): Die Waldgeschichte Ostholsteins und der zeitliche Verlauf der postglazialen Transgression an der holsteinischen Ostseeküste. - Ber. Dt. Bot. Ges., 66, 3: 151-166; Berlin.

SChÜтRUMPF, R. (1952): Die pollenanalytische Horizontierung der Knochenfunde von Barsbek, Kreis Plön, s. Guenther et al.: 38-43.

- (1972): Die Stratigraphie und pollenanalytischen Ergebnisse der Ausgrabung des Ellerbekzeitlichen Wohnplatzes Rosenhof (Ostholstein). - Archäolog. Korrespondenzblatt, 2: 9-16; Mainz.

- (1976): Stratigraphisch-pollenanalytische Untersuchungen am neolithischen Fundplatz Rosenhof zur relativen Datierung und Bestimmung des Ostseemeeresspiegels zur Zeit der Besiedlung. - Eiszeitalter u. Gegenwart, 27: 195; Öhringen/Württ.

Schwabedissen, H. (1972): Rosenhof (Ostholstein), ein Ellerbek-Wohnplatz am einstigen Ostseeufer. - Archäol. Korrespondenzblatt 2: 1-8, Mainz.

TAPFER, E. (1940): Meeresgeschichte der Kieler und Lübecker Bucht im Postglazial. - Geol. d. Meere u. Binnengewässer, 4: 113-244; Berlin.

Truelsen, G. (1973): Die Wasserstandsänderungen in der südwestlichen Ostsee während der letzten 100 Jahre. - Realschullehrerexamensarbeit (angefertigt bei H. KLUG), Kiel. - [Unveröff.].

Voss, F. (1967): Die morphologische Entwicklung der Schleimündung. - Hamburger Geogr. Studien, 20 (Diss. Hamburg 1965): ०—o; Hamburg.

- (1968): Junge Erdkrustenbewegungen im Raume der Eckernförder Bucht. - Mitt. d. Geogr. Ges. Hamburg, 57: 97-189; Hamburg.

- (1970): Der Einfluß des jüngsten Transgressionsablaufes auf die Küstenentwicklung der Geltinger Birk im Nordteil der westlichen Ostsee. - Die Küste, 20: 101-113; Heide.

- (1972): Neue Ergebnisse zur relativen Verschiebung zwischen Land und Meer im Raum der westlichen Ostsee. - Z. Geomorph., NF, Suppl. Bd. 14: 150-168; Berlin.

- (1973): Geomorphologische und archäologische Untersuchungen im Mündungsgebiet der Langballigau an der Flensburger Förde. - Archäolog. Korrespondenzblatt, 3, 1:123-127; Mainz.

- M. Müller-Wille \& E. W. RAABe (1973): Das Höftland von Langballigau an der Flensburger Förde. - Offa, 30: 60-132; Neumünster.

Willoomm, H. \& H. ErLEnkeuser (1969): University of Kiel Radiocarbon Measurements IV. Radiocarbon, 11, 2: 423-429; New Haven.

Manuskript eingegangen am 6. 2. 1980. 